\title{
Rasgo conciencia y actitud hacia la tesis en universitarios de una sociedad científica
}

\author{
Oscar Javier Mamani-Benito ${ }^{1}$, Edison Effer Apaza Tarqui ${ }^{2}$ \\ Universidad Peruana Unión-Perǘ1,2, Universidad Privada San Carlos-Perü ${ }^{1}$
}

\begin{abstract}
Con el objetivo de determinar si el rasgo conciencia de la personalidad es un buen predictor de la actitud hacia la tesis, se realizó un estudio asociativo predictivo con una población de 83 universitarios de una sociedad científica a quienes se les aplicó la subescala factor conciencia del inventario de personalidad NEO PI-R y la escala de actitud hacia la realización de una tesis. Los resultados evidencian que las facetas competencia, sentido del deber y reflexión son las que predicen significativamente la actitud hacia la tesis universitaria. Así mismo, se observa una asociación directa a nivel moderado entre ambas variables. Se concluye que la predominancia del rasgo conciencia en el perfil de personalidad de universitarios involucrados en grupos de investigación predice actitudes favorables hacia la elaboración de trabajos de grado.
\end{abstract}

Palabras clave: personalidad, rasgo conciencia, actitudes hacia la investigación, tesis.

\section{Conscientiousness and attitude towards the thesis in university students of a scientific society}

In order to determine if the Conscientiousness trait of personality is a good predictor of the attitude towards the thesis, a predictive correlative study was carried out with a population of 83 university students of a Scientific Society. The conscientiousness sub-scale of the NEO PI-R personality inventory and the attitude scale towards the realization of a thesis were applied. The results show that the facets of competence, sense of duty and reflection are what significantly predict the attitude towards the university thesis; likewise, a direct and moderate association is observed between both variables. The predominance of the conscientiousness trait in the personality profile of university students involved in research groups predicts favorable attitudes towards the elaboration of degree work.

Keywords: personality, conscienctiousness, attitudes towards research, thesis.

Traço de consciência e atitude em relaçáo à tese em estudantes universitários de uma sociedade científica

A fim de determinar se o traço de consciência da personalidade é um bom preditor da atitude em relação à tese, foi realizado um estudo associativo preditivo com uma amostra

1 Magíster en Educación. Profesor en la Escuela de Posgrado y docente investigador en la Universidad Peruana Unión y Universidad Privada San Carlos, Perú. Investigador certificado por Concytec. Dirección postal: Avenida Simón Bolívar 1019, Puno (Perú). Contacto: oscar.mb@ upeu.edu.pe; oscar.mamani@upsc.edu.pe. ORCID: https://orcid.org/0000-0002-9818-2601

2 Ingeniero estadístico e informático. Docente en la Universidad Peruana Unión. Dirección postal: 15464 Lima, Lima, Lurigacho - Universidad Peruana Unión. Contacto: effer@upeu. edu.pe. ORCID: https://orcid.org/0000-0002-6520-3795 
de 83 estudantes universitários de uma sociedade científica a quem foi aplicado o fator de subescala consciência do inventário de personalidade NEO PI-R e a escala de atitude para a realização de uma tese. Os resultados mostram que as facetas de competência, senso de dever e reflexão são o que predizem significativamente a atitude em relação à tese universitária, assim como, uma associação direta é observada em um nível moderado entre as duas variáveis. Conclui-se que a predominância do traço de consciência no perfil de personalidade dos universitários envolvidos em grupos de pesquisa prediz atitudes favoráveis à elaboraçáo de trabalhos de graduação.

Palavras chave: personalidade, traço de consciência; atitudes em relação à pesquisa, tese.

\section{Conscience des facteurs et attitude envers la thèse chez les étudiants universitaires d'une société scientifique}

Afin de déterminer si le trait de conscience de la personnalité est un bon prédicteur de l'attitude envers la thèse, une étude associative prédictive a été réalisée avec une population de 83 étudiants universitaires d'une société scientifique à laquelle le facteur de conscience de sous-échelle a été appliqué. De l'inventaire de la personnalité NEO PI-R et l'échelle d'attitude envers la réalisation d'une thèse. Les résultats montrent que ce sont les facettes de la compétence, du sens du devoir et de la réflexion qui prédisent de manière significative l'attitude à l'égard de la thèse de l'université. De même, une association directe est observée à un niveau modéré entre les deux variables. Nous concluons que la prédominance du trait de conscience dans le profil de personnalité des étudiants universitaires impliqués dans des groupes de recherche prédit des attitudes favorables à l'égard de l'élaboration de diplômes. Mots-clés: personnalité, trait de conscience, attitudes envers la recherche, thèse. 
Vivimos en tiempos donde el ejercicio de la investigación científica está causando gran interés fuera del contexto de las universidades. Actualmente no son unos cuantos académicos o entidades gubernamentales que exigen la producción de nuevo conocimiento con la finalidad de cumplir estándares de acreditación o posicionamiento en los rankings mundiales, más bien, hoy con mayor énfasis ejercer investigación se ha convertido en una necesidad, sobre todo para aquellos países que se proponen impulsar una transición hacia una economía menos dependiente de los recursos naturales (Organización de las Naciones Unidas para la Educación, la Ciencia y la Cultura [Unesco], 2015).

Al tratar de configurar el panorama actual de la producción científica en el mundo, encontramos que Estados Unidos y los países de la Unión Europea generan poco más del $50 \%$ del nuevo conocimiento, así también, se resalta el ascenso de China, pues en solo cinco años ha pasado de aportar del 5\% al 20\% de la producción científica mundial (Unesco, 2015). Al respecto, se estima que la producción científica mundial mantiene un incremento anual del 3\% y una cantidad de publicaciones que se duplica cada 24 años. Sin embargo, este crecimiento no es homogéneo en todas las regiones, pues entre 1996 y 2014 América Latina solo logró aportar cerca del 3.3\% y Perú 1.04\% a la región (Robles-Alfaro, Vela-Alfaro, Huapaya-Huertas \& ChacónTorrico, 2015). Estas cifras confirman la enorme brecha existente entre países desarollados con países donde aún se lucha por fortalecer los sistemas de educación superior, mejorar la calidad de investigación y sobre todo incrementar la inversión en $\mathrm{I}+\mathrm{D}+\mathrm{i}$.

En el caso de la realidad peruana, tener conciencia de la problemática de la producción científica en el país lleva a una reflexión obligada respecto a las causas y factores involucrados (Pereyra-Elías, HuacchoRojas, Taype-Rondán, Mejía \& Mayta-Tristán, 2014). Ya con antelación, Alarco, Changlio-Calle y Cahuana-Salazar (2017) habían evidenciado 
que el Perú fue considerado dentro de América del Sur como el país que tuvo la más baja proyección de producción científica en el año 2015. Es por ello, que en los últimos años el foco de atención se ha centrado en el estado actual del sistema educativo universitario y la falta de promoción de la investigación desde las universidades. Aunado a esto, investigadores como Taype-Rondan et al. (2014) fueron más precisos al referir que uno de los factores que incide en esta problemática, al igual que en la mayoría de países latinoamericanos, obedece a un tema de carácter actitudinal pues se ha hecho evidente que existe una escasa participación estudiantil para el ejercicio de la investigación científica. En respuesta a este hecho, las Sociedades Científicas se perfilan como la mejor estrategia para impulsar la investigación desde la universidad.

En este punto, cabe resaltar que una de las principales fuentes para la publicación científica son las tesis de grado (Mayta-Tristán, 2016), no obstante, algunos reportes hallados en la literatura científica revelan la existencia de una tasa muy baja de publicación de este tipo de trabajos en revistas indexadas (Atamari-Anahui, Roque-Roque, Robles-Mendoza, Nina \& Falcón-Huancahuiri, 2015; Castro, Sihuay-Torres \& Pérez-Jiménez, 2017; Taype-Rondán, Carbajal-Castro, ArrunáteguiSalas \& Chambi-Torres, 2012; Ticse, Ygreda \& Samalvides, 2014). Para los expertos, claramente esta sería una de las principales causas que estaría obstruyendo el impulso que requiere la producción científica en nuestro país. Sin embargo, existe un grupo de estudiantes y docentes que sí han logrado generar resultados relevantes, y son precisamente estos, los que revelan algunas características asociadas al involucramiento con la investigación y la producción científica estudiantil. Por ejemplo, un estudio realizado por Mejía, Inga-Berrospi, y Mayta-Tristán (2014) respecto a la motivación de 98 tesistas de una universidad de Lima en proceso de elaborar su tesis, entre estudiantes y egresados, reveló, que el $82.7 \%$ evidenciaba como una de sus motivaciones principales el hecho de que disfrute investigar. En la misma línea, otro estudio reciente realizado por Aiquipa, Ramos, Curay y Guizaldo (2018) evidenció que uno de los factores implicados para realizar o no una tesis en estudiantes universitarios fue el psicológico, y dentro de este aspecto resaltan el 
sistema de creencias y los rasgos de personalidad. Precisamente, este último, según la investigación mencionada, es el que explica cómo la evitación del esfuerzo y una tendencia al inmediatismo estarían influyendo negativamente en el interés por investigar $y$, en consecuencia, un déficit de habilidades para la investigación.

En cuanto a este interés y motivación necesarios para el ejercicio de la investigación científica, existe literatura sobre diversas iniciativas de investigación que se centran en descubrir los factores relacionados al éxito académico en el contexto universitario (Borracci et al., 2014; Mills, Heyworth, Rosenwax, Carr \& Rosenberg, 2009). Estos estudios claramente evidencian que la personalidad humana está fuertemente vinculada a procesos internos de los individuos y sus motivaciones en el campo académico, que, en consecuencia, generan un adecuado rendimiento académico y un importante grado de motivación de logro ante tareas y exigencias como las que demandan aquellas que implican rigor científico (Al-Naggar et al., 2015; Chamorro-Premuzic \& Furnham, 2008; Eyong, David \& Umoh, 2014; Hakimi, Hejazi \& Lavasani, 2011; Mamani \& Quinteros, 2014; Sobowale, Ham, Curlin \& Yoon, 2017; Tijdink et al., 2016; Vedel \& Poropat, 2017).

El estudio de la personalidad humana ha sido, a través de los años, uno de los temas de investigación más importantes para la comprensión del ser humano y su comportamiento. Las teorías que gozan de gran aceptación defienden el hecho que tanto los pensamientos, sentimientos y las acciones son modulados por mecanismos internos, estables y consistentes a través del tiempo. Precisamente, uno de estos modelos teóricos viene a ser el propuesto por Paul Costa y Robert McCrae (Romero, Ángeles, Gómez \& Sobral, 2002), quienes lo nombraron: El modelo de los Cinco Factores. En esta teoría, los autores proponen que todos los individuos pueden ser caracterizados en base a cinco grandes rasgos de la personalidad: neuroticismo, extraversión, agradabilidad, apertura y conciencia. Especialmente este último, se ha convertido en la variable de mayor interés para los investigadores que buscan explicar el éxito académico en el ámbito educativo. Esto debido a que el hecho de que un estudiante alcance con excelencia determinadas metas vendría a ser 
el producto de un significativo grado de necesidad de logro, persistencia, competencia y motivación de una persona, tal como lo evidencian sujetos con altos niveles del rasgo conciencia en su perfil de personalidad.

Proponer esta asociación como una causalidad latente tiene asidero a la luz de los hallazgos encontrados en la literatura científica tanto a nivel nacional como internacional. En el Perú, empezando por aquellas que vinculan la personalidad y sus cinco factores con variables académicas, sobresale el trabajo de Cassaretto, Niño de Guzmán y Calderón (2003), quienes concluyeron que el rendimiento académico fue mejor explicado por el rasgo conciencia, específicamente las faceras aspiraciones de logro y reflexión. Así también, respecto a iniciativas que vinculan netamente los rasgos de personalidad con la actitud hacia la tesis en universitarios, se resalta el trabajo realizado por Mamani y Quinteros (2014), quienes también concluyeron que cuanto mayor predominancia tiene el rasgo conciencia en el perfil de personalidad de los estudiantes, mayores actitudes favorables hacia la tesis son reveladas. Por otra parte, en el ámbito internacional, donde aparecen la mayor cantidad de estudios que avalan que la personalidad, es evidente que este rasgo ha demostrado ser un predictor para determinar un buen desempeño y éxito académico (Al-Naggar et al., 2015; Chamorro-Premuzic \& Furnham, 2008; Eyong, David \& Umoh, 2014; Hakimi, Hejazi \& Lavasani, 2011; Sobowale, Ham, Curlin \& Yoon, 2017; Tijdink et al., 2016; Vedel \& Poropat, 2017), resaltando algunos como el realizado por Hong y Li (2011), quienes concluyeron que el rasgo conciencia es un predictor significativo de actitudes favorables hacia la ciencia en estudiantes de educación secundaria. Así también, el realizado por Malykh (2017), el cual concluye que el único rasgo de personalidad que tiene la capacidad de explicar las diferencias individuales respecto al logro académico en una población de estudiantes de secundaria, fue el denominado rasgo o factor conciencia.

Por último, respecto a la realidad peruana en producción científica estudiantil en los últimos años, la literatura científica ha revelado el rol de las sociedades científicas como gestoras de producción de nuevo conocimiento en el ámbito universitario (Vargas, 2018). Los denominados 
grupos para la investigación están conformados casi en su totalidad por estudiantes con intereses a fines de investigación (Cvetkovic-Vega, IngaBerrospi \& Mestas, 2016). Al respecto, los estudios realizados, así como la opinión de autores en el tema, refieren que pertenecer a una sociedad científica incrementa la probabilidad de publicar un artículo científico (Mejía, Valladares-Garrido, Almanza-Mio \& Benites-Gamboa, 2018; Taype-Rondán, Lajo-Aurazo, Gutiérrez-Brown, Zamalloa-Masías \& Saldaña-Gonzales, 2002).

Por lo tanto, en función de probar una hipótesis que implica asumir que la predominancia del rasgo conciencia en el perfil de personalidad, incrementa significativamente la probabilidad de generar actitudes favorables hacia la realización de trabajos de grado en estudiantes que pertenecen a grupos de investigación como es el caso de las sociedades científicas, es que surge la pregunta de investigación: ¡es el rasgo conciencia un buen predictor de las actitudes favorables hacia la tesis en universitarios? A consecuencia, en el presente estudio se tienen como objetivos determinar si este es un predictor de la actitud hacia la realización de una tesis, así como explorar las relaciones entre las dimensiones de conciencia con la actitud hacia realización de una tesis.

\section{Método}

\section{Participantes}

Se trabajó con un grupo de 83 universitarios miembros activos de la sociedad científica de estudiantes de una universidad privada de Juliaca, Perú. Estos pertenecían a cuatro facultades: 22.8\% de Ciencias de la Salud (19), 21.6\% de Ciencias Empresariales (18), 10.8\% de Ciencias Humanas y Educación (9) y 44.5\% de Ingeniería y Arquitectura (37), siendo el $40.9 \%$ varones (34) y 59.1\% mujeres (49). La totalidad de los participantes accedieron a entregar sus datos para fines de la investigación, los mismos fueron tratados en el anonimato para proteger su información personal, cumpliendo con las disposiciones éticas que rigen a esta investigación. 


\section{Medición}

Rasgo conciencia de la personalidad. Para la medición de esta variable se recurrió al inventario de personalidad NEO PI-R de McCrae y Costa (2004), específicamente se hizo uso de la subescala que mide solo el factor conciencia de la personalidad. Este inventario está compuesto por 48 ítems que miden seis facetas: competencia, orden, sentido del deber, aspiración de logro, autodisciplina y reflexión, y tiene como opciones de respuesta alternativas en cinco niveles tipo Likert; 1 = totalmente en desacuerdo (TD), 2 = en desacuerdo (D), 3 = neutral $(\mathrm{N}), 4=\mathrm{de}$ acuerdo (A), y 5 = totalmente de acuerdo (TA). El instrumento como escala total cuenta con una adaptación al Perú realizada por Cassaretto (2009), sin embargo, para objetivos de la investigación, se procedió a validar solo el contenido de la subescala.

Fueron tres especialistas en el ámbito de la psicología clínica en calidad de jueces y, que según el formato validación, valoraron, en una escala de 1 al 100 (.1 a 1), la pertinencia de los ítems, la claridad en cuanto al lenguaje y comprensión de los mismos, su congruencia en relación a la faceta evaluada, y la coherencia que mantienen con el constructo de la personalidad. Una vez obtenidos los puntajes se pasó a promediar cada uno de ellos obteniendo un índice de acuerdo total $(\mathrm{I} / \mathrm{A}) \mathrm{de}=.99$. Posterior a ello, se pasó a determinar su consistencia interna mediante una prueba piloto aplicada en 30 alumnos de primer año de una universidad privada de Juliaca, obteniendo un coeficiente $\alpha$ de .83 . Con estos valores se asume que el instrumento es válido y confiable.

Actitud hacia la realización de una tesis. Para la medición de esta variable se recurrió a la prueba de Disposición para la Realización de una tesis elaborada por Ruiz (2005). Esta escala consta de 44 ítems distribuidos para la evaluación de cuatro factores: importancia en la formación académica, facilidades académicas, utilidad futura y confianza académica para la tesis, teniendo como opciones de respuesta niveles tipo Likert: 4 = de acuerdo (DA), 3 = indeciso (I), 2 = en desacuerdo (ED) y 1 = totalmente en desacuerdo (TD). Al igual que en el caso anterior, se procedió a validar el contenido por tres expertos en calidad de jueces, especialistas en metodología de la investigación y autores de 
artículos científicos en revistas indexadas. Así como en el caso de la variable personalidad, el formato de validación implicó la valoración en una escala de 1 al 100, la pertinencia, coherencia, congruencia y claridad de los ítems; obteniéndose $\mathrm{I} / \mathrm{A}=.90$. Posteriormente, se pasó a determinar la consistencia interna con la misma población para la prueba piloto obteniendo un coeficiente $\alpha$ de .81 , con lo cual se concluye que el instrumento es válido y confiable.

\section{Procedimiento}

Antes de proceder a la recolección de datos se realizaron las gestiones necesarias para obtener la autorización del comité de ética de la universidad en cuestión, en este caso se recurrió a la sede principal ubicada en la ciudad de Lima, Perú. Para estos trámites se presentó la documentación necesaria según los formatos establecidos por la Dirección General de Investigación. Una vez recibido el documento de aprobación, se procedió a organizar la aplicación de ambos instrumentos en el evento denominado VII Jornada Científica de Estudiantes, llevada a cabo en junio del año 2017. Para tal motivó se solicitó el uso de un ambiente donde se congregó a todas las directivas de las sociedades científicas de todas las facultades. Seguidamente, se detalló el consentimiento informado a cada participante y brevemente los objetivos del estudio, poniendo énfasis en la confidencialidad de los datos, así como su participación voluntaria. Luego de aproximadamente 45 minutos se obtuvo las encuestas debidamente llenadas y posterior a ello se procedió a su calificación.

\section{Análisis de datos}

Los datos fueron codificados e introducidos en el programa estadístico SPSS versión 22.0. Se calcularon las frecuencias y porcentajes de cada variable. Para analizar el valor predictivo del rasgo conciencia sobre la actitud hacia la tesis se utilizó el análisis de regresión lineal múltiple. Asimismo, para estudiar la relación entre las variables y sus dimensiones, en concordancia a la prueba de normalidad de Kolmogorov-Smirnov, se utilizó una prueba no paramétrica como es el coeficiente de correlación Tau B (Tb) de Kendall. 


\section{Resultados}

\section{Predominancia del rasgo conciencia}

Respecto a la predominancia del rasgo conciencia en el perfil de personalidad de los universitarios que pertenecen a la sociedad científica de estudiantes, según la Tabla 1, se halló que este factor predomina a un nivel alto en el $43.4 \%$ de los encuestados, a nivel moderado en el $42.2 \%$ y a nivel bajo en el $14.5 \%$. Por otra parte, en cuanto a las facetas que la componen, se observa una predominancia alta en sentido del deber (54.2\%) y aspiraciones de logro (61.4\%), mientras que se aprecia una predominancia moderada en competencia $(37.3 \%)$, orden $(54.2 \%)$ y autodisciplina $(47.0 \%)$. Finalmente, se observa una predominancia baja en reflexión (38.6\%) y competencia $(30.1 \%)$.

\section{Tabla 1}

Nivel de predominancia del rasgo conciencia en la población de estudio

\begin{tabular}{lcccccc}
\hline & \multicolumn{2}{c}{ Bajo } & \multicolumn{2}{c}{ Moderado } & \multicolumn{2}{c}{ Alto } \\
\cline { 2 - 7 } & $\%$ & $\mathrm{f}$ & $\%$ & $\mathrm{f}$ & $\%$ & $\mathrm{f}$ \\
\hline Rasgo conciencia & 14.5 & 12 & 42.2 & 35 & 43.4 & 36 \\
Competencia & 30.1 & 25 & 37.3 & 31 & 32.5 & 27 \\
Orden & 14.5 & 12 & 54.2 & 45 & 31.3 & 26 \\
Sentido del deber & 6.0 & 5 & 39.8 & 33 & 54.2 & 45 \\
Aspiraciones de logro & 10.8 & 9 & 27.7 & 23 & 61.4 & 51 \\
Autodisciplina & 25.3 & 21 & 47.0 & 39 & 27.7 & 23 \\
Reflexión & 38.6 & 32 & 36.1 & 30 & 25.3 & 21 \\
\hline
\end{tabular}

\section{Actitud hacia la realización de una tesis}

Respecto a las actitudes manifiestas por participantes, según la Tabla 2, el $48.2 \%$ de los encuestados demuestra actitudes muy favorables hacia la realización de una tesis; $34.9 \%$, actitudes medianamente 
favorables y; solo $16.9 \%$, actitudes desfavorables. En cuanto a las dimensiones, se observa una predominancia de actitudes medianamente favorables en formación académica (56.6\%) y utilidad futura (50.6), mientras que existe cierta predominancia de actitudes desfavorables en facilidades académicas $(57.8 \%)$ y confianza académica para la tesis (44.6\%).

\section{Tabla 2}

Actitudes hacia la realización de una tesis en la población de estudio

\begin{tabular}{lcccccc}
\hline & \multicolumn{2}{c}{ Desfavorable } & \multicolumn{2}{c}{ Medianamente favorable } & \multicolumn{2}{c}{ Muy favorable } \\
\cline { 2 - 7 } & $\%$ & $\mathrm{f}$ & $\%$ & $\mathrm{f}$ & $\%$ & $\mathrm{f}$ \\
\hline Actitud hacia la tesis & 16.9 & 14 & 34.9 & 29 & 48.2 & 40 \\
Formación académica & 12.0 & 10 & 56.6 & 47 & 31.3 & 26 \\
Facilidades académicas & 57.8 & 48 & 34.9 & 29 & 7.2 & 6 \\
Utilidad futura & 0.0 & 0 & 50.6 & 42 & 49.4 & 41 \\
Confianza académica & 44.6 & 37 & 43.4 & 36 & 12.0 & 10 \\
\hline
\end{tabular}

\section{Análisis de correlación}

Los valores obtenidos en la Tabla 3 son indicativos de la existencia de una correlación positiva, de magnitud moderada y estadísticamente significativa $(\mathrm{Tb}=.56, p<.01)$ entre el rasgo conciencia de la personalidad y la actitud hacia la realización de una tesis en el pregrado. Así también, se observan correlaciones positivas a nivel moderado con las dimensiones competencia $(\mathrm{Tb}=.55, p<.01)$, sentido del deber $(\mathrm{Tb}=.51, p=<.01)$, autodisciplina $(\mathrm{Tb}=.52, p=<.01)$, y reflexión $(\mathrm{Tb}=.56, p<.01)$; mientras que las correlaciones son de magnitud baja con la dimensión orden $(\mathrm{Tb}=.37, p<.01)$ y aspiraciones de logro $(\mathrm{Tb}=.28, p<.01)$. 


\section{Tabla 3}

Matriz de correlaciones entre las variables rasgo conciencia de la personalidad y actitud hacia la realización de una tesis

\begin{tabular}{lccccccc}
\hline & $\mathrm{RC}$ & $\mathrm{C}$ & $\mathrm{O}$ & $\mathrm{SD}$ & $\mathrm{AL}$ & $\mathrm{A}$ & $\mathrm{R}$ \\
\hline $\mathrm{ART}$ & $.56^{* *}$ & $.55^{* *}$ & $.37^{* *}$ & $.51^{* *}$ & $.28^{* *}$ & $.52^{* *}$ & $.51^{* *}$ \\
$\mathrm{RC}$ & & $.58^{* *}$ & $.65^{* *}$ & $.60^{* *}$ & $.42^{* *}$ & $.68^{* *}$ & $.65^{* *}$ \\
$\mathrm{C}$ & & & $.27^{* *}$ & $.47^{* *}$ & .09 & $.54^{* *}$ & $.54^{* *}$ \\
$\mathrm{O}$ & & & & $.48^{* *}$ & $.45^{* *}$ & $.48^{* *}$ & $.48^{* *}$ \\
$\mathrm{SD}$ & & & & & $.27^{* *}$ & $.36^{* *}$ & $.27^{* *}$ \\
$\mathrm{AL}$ & & & & & & $.37^{* *}$ & $.39^{* *}$ \\
$\mathrm{~A}$ & & & & & & & $.68^{* *}$ \\
\hline
\end{tabular}

${ }^{* *}$ Valor de $p<.01$.

ART: actitud hacia la realización de una tesis; RC: rasgo conciencia; C: competencia, O: orden; SD: sentido del deber; AL: aspiraciones de logro; A: autodisciplina; R: reflexión.

\section{Análisis de regresión lineal múltiple}

En la Tabla 4 se aprecia el modelo de regresión lineal múltiple, el cual explica el efecto de los indicadores de la variable rasgo de conciencia en la actitud hacia la realización de una tesis. Al asumir que los indicadores utilizados son cuantitativos y luego de contrastar los supuestos estadísticos para validar el modelo esperado, se halló que estos demuestran que tres dimensiones: competencia, sentido del deber y reflexión, explican la variabilidad de la actitud hacia la tesis en un $63.5 \%$. Cabe recalcar, que para lograr este nivel de predicción se tuvo que quitar tres indicadores, que fueron orden, aspiraciones de logro y autodisciplina, los cuales obtuvieron una significancia $p>.05$ y un $R^{2}$ ajustado de .62. En tal contexto, por principio de parsimonia se configuró un modelo que explique con el menor número de indicadores o variables, y los tres mencionados lo demuestran al tener un valor $p$ menor al $.05(p<.05)$. Finalmente, por el resultado de la prueba $t$ de Student, se puede asumir que la dimensión sentido del deber viene a ser el predictor más importante $(t=3.224, p<.05)$, seguido de reflexión $(t=3.017, p<.05)$ y por último competencia $(t=2.798, p<.05)$. 


\section{Tabla 4}

Coeficientes de regresión lineal múltiple en base al efecto de los indicadores del rasgo de conciencia en la actitud hacia la tesis

\begin{tabular}{lcccc}
\hline Modelo: & \multicolumn{2}{c}{ Coeficientes no estandarizados } & & \\
\cline { 2 - 3 }$R^{2}$ ajustado $=.635$ & $B$ & Error estándar & & \\
\hline (Constante) & 64.955 & 2.634 & 24.660 & .000 \\
Competencia & .361 & .129 & 2.798 & .006 \\
Sentido del deber & .343 & .106 & 3.224 & .002 \\
Reflexión & .391 & .129 & 3.017 & .003 \\
\hline
\end{tabular}

Variable dependiente: Actitud

\section{Supuestos de normalidad}

En la Tabla 5 se presentan los resultados de los supuestos de la media de los errores. Al probar la normalidad de los errores los resultados del $p$ valor para los valores no estandarizados y estandarizados son iguales a $.01(p>.05)$, lo que indica que los datos no provienen de una distribución normal. Sin embargo, también es necesario aclarar que para un análisis de regresión son funcionales tanto distribuciones normales como no normales.

\section{Tabla 5}

Supuestos media igual a cero de los errores y normalidad en los errores

\begin{tabular}{llcc}
\hline & & $\begin{array}{c}\text { Errores no } \\
\text { estandarizados }\end{array}$ & $\begin{array}{c}\text { Errores } \\
\text { estandarizados }\end{array}$ \\
\hline $\mathrm{N}$ & Media & 83 & 83 \\
\multirow{2}{*}{ Parámetros normales } & Desviación estándar & 2.783 & .000 \\
Kolmogorov-Smirnov & & .000 & .982 \\
$\mathrm{p}$ & & .01 & .114 \\
\hline
\end{tabular}


En la misma línea, en la Tabla 6 se presentan dos resultados, los cuales muestran el supuesto de multicolinealidad, para el cual la medición se genera por medio del Factor de Inflación de Varianza (VIF), que en este caso son menores a 10 puntos. Esto indica que las correlaciones entre las variables independientes son menores al $90 \%$, mientras que el estadístico de Durbin-Watson, que mide la autocorrelación de errores, demuestra que el resultado del estadístico es de 1.704, lo cual indica que esta entre los parámetros de 1.5 a 2.5 , y esto demuestra que no existe autocorrelación entre los errores.

\section{Tabla 6}

Supuestos de multicolinealidad y autocorrelación de errores

\begin{tabular}{lccc}
\hline \multirow{2}{*}{ Modelo } & \multicolumn{2}{c}{ Estadísticas de colinealidad } & \multirow{2}{*}{$\begin{array}{l}\text { Durbin- } \\
\text { Watson }\end{array}$} \\
\cline { 2 - 3 } & Tolerancia & VIF & \\
\hline Competencia & .323 & 3.097 & \\
Sentido del deber & .587 & 1.703 & 1.704 \\
Reflexión & .413 & 2.421 & \\
\hline
\end{tabular}

\section{Discusión}

El presente estudio tuvo como objetivo determinar si el rasgo conciencia de la personalidad es un predictor estadísticamente significativo de la actitud hacia la realización de una tesis, en un grupo de universitarios que pertenecen a una sociedad científica. De acuerdo a los resultados, existe un predominio del rasgo conciencia en el perfil de personalidad de los encuestados, así como actitudes favorables hacia la realización de una tesis. Por otro lado, el modelo de regresión lineal múltiple muestra que son tres facetas: competencia, sentido del deber y reflexión, las que explican significativamente la variabilidad de la actitud hacia la tesis. Así mismo, el coeficiente de correlación indica asociaciones positivas de magnitud moderada entre la variable conciencia y sus dimensiones, con la variable actitud. Similares resultados, aunque 
no en profundidad como la presente, fueron hallados por Mamani y Quinteros (2014) quienes luego de encuestar a una población de estudiantes de psicología de una universidad privada encontraron que un 64.7\% evidenció una actitud entre moderada a muy favorable hacia la tesis universitaria. Además, se halló una correlación significativa entre el rasgo conciencia y la actitud hacia la tesis universitaria. Sin embargo, el perfil de personalidad de los encuestados no demostró predominio del rasgo conciencia. Esta diferencia, Podría relacionarse al hecho de que en la investigación citada no se tenía como criterio de inclusión pertenecer a una sociedad científica.

Los hallazgos de la presente investigación, orientan a asumir que el perfil de personalidad de universitarios con interés de aprender investigación en grupos como las sociedades científicas, se caracteriza según el modelo de los cinco factores expuesto en Casarretto (2009), por tener un marcado sentimiento de capacidad y eficacia personal (competencia), preocupación por el orden y la organización (orden), tendencia al cumplimiento estricto de las obligaciones personales (sentido del deber), determinación de metas y esfuerzo dedicado para el logro de estas (aspiraciones de logro), habilidad para planificar y llevar a cabo una tarea a pesar del aburrimiento y otras distracciones (Autodisciplina), y, tendencia a pensar cuidadosamente antes de actuar (Reflexión). Además, el hecho de que más del 50\% de la población de encuestados demostró actitudes entre medianamente favorables a muy favorables hacia la tesis, también demuestra que este tipo de estudiantes se muestran predispuestos a enfrentar el proceso de elaboración de un trabajo de grado. Sobre estos hechos, una investigación preliminar realizada por Toro-Huamanchumo, Failoc-Rojas y Díaz-Vélez (2015) en estudiantes de medicina ya había logrado comprobar que los estudiantes que pertenecen a grupos de investigación evidencian una mayor capacidad de aprender con facilidad metodología de la investigación. Así mismo, estos mostrarían una adecuada actitud hacia la investigación científica, lo cual hace suponer que es precisamente su estructura de personalidad orientada a la necesidad logro y autoeficacia personal la que los lleva a asumir tareas exigentes con responsabilidad. 
Respecto a otros hallazgos, se muestra, en primera instancia, la existencia de una predominancia de actitudes desfavorables en las dimensiones facilidades académicas y confianza académica. Sobre este hecho, teniendo en cuenta que estas tienen que ver con los factores institucionales, similares resultados fueron encontrados por Alarco, Aguirre-Cuadros, Aliaga-Chávez y Alvarez-Andrade (2010) quienes también hallaron que los estudiantes perciben como obstáculos los trámites engorrosos y la falta de docentes con producción científica. De igual modo, como segundo punto, es ciertamente preocupante haber encontrado que un 56.6\% evidenció actitudes medianamente favorables en la dimensión formación académica, por lo que se supone que si uno recibe una buena formación entonces debería sentir confianza para enfrentar una tesis. Sin embargo, es necesario aclarar que la calidad de la formación recibida no siempre es suficiente cuando existe de por medio creencias irracionales acerca de la tesis (Aiquipa, Ramos, Curay \& Guizaldo, 2018) y experiencias negativas producto de una interacción con docentes de investigación que no demuestran competencias investigativas para la producción científica.

El hecho de haber encontrado que el rasgo conciencia, especialmente sus facetas competencia, sentido del deber y reflexión, tengan la capacidad de predecir la actitud hacia la tesis, es un resultado acorde a la literatura científica. Esto es argumentado en estudios anteriores como el realizado por Cupani, Garrido y Tavella (2013), quienes mencionan que gran parte de investigaciones que tuvieron el objetivo de explorar la relación entre la personalidad y el éxito académico en universitarios, han logrado sustentar el valor predictivo del rasgo conciencia como un factor influyente para demostrar un adecuado rendimiento académico. De igual modo, otros hallazgos concluyeron que existe un grado de asociación muy significativo entre los rasgos de personalidad y variables que tienen que ver con el éxito en el ámbito académico (Hakimi, Hejazi \& Lavasani, 2011; Hazrati-Viari, Tayarani \& Saeed, 2012; Komarraju \& Karau, 2005; Caprara, Vecchione, Alessandri, Gerbino \& Barbaranelli, 2011; Laidra, Pullmann \& Allik, 2007; Smidt, 2015; Verešová, 2015; Vern, 1958). 
A diferencia de estudios anteriores, la presente investigación logró demostrar esta dinámica predictiva en una población que hasta la actualidad no se había tomado en cuenta. Esto claramente orienta a repensar los procesos de investigación en el pregrado, sobre todo la investigación formativa, pues queda demostrado que quienes se caracterizan por ser escrupulosos, ordenados, estrictos en el cumplimiento de las tareas, así como reflexivos y cuidadosos en la resolución de problemas (McCrae \& Costa, 1987) están en condiciones de enfrentar con éxito no solo la realización de una tesis, que ya implica un proceso complejo, que además de aspectos cognitivos y metodológicos también involucra la intervención de factores personales y psicológicos (Rietveldt \& Vera, 2012), sino también en condiciones de formarse como futuros investigadores que el pais tanto necesita.

En conclusión, a la luz de los resultados antes discutidos, una predominancia significativa del rasgo conciencia en el perfil de personalidad de los universitarios que pertenecen a grupos de investigación permite predecir adecuadamente actitudes favorables hacia la realización de una tesis, lo cual permite aceptar la hipótesis de investigación planteada. Además, los hallazgos fortalecen lo hasta ahora expuesto en la literatura científica respecto a la capacidad que tiene este rasgo para predecir el éxito académico frente a exigencias en el ámbito universitario.

Respecto a las limitaciones, la principal recae sobre el tamańo de la población, pues la cantidad de la presente no permite una generalización más que solo a la sociedad científica de la universidad involucrada. Ante ello, se recomienda que futuras investigaciones incluyan poblaciones representativas por tipo de universidad (estatal o privada), tomando en cuenta sociedades científicas ya establecidas y con gran trayectoria como es el caso de las de medicina. Se recomienda también, la réplica del presente estudio en otras universidades para confirmar el valor predictivo y las características de personalidad de universitarios que pertenecen a grupos de investigación. 


\section{Referencias}

Aiquipa, J., Ramos, C., Curay, R. \& Guizaldo, L. (2018). Factores implicados para realizar o no realizar tesis en estudiantes de psicología. Propositos y Representaciones, 6(1), 2-26. https://doi.org/ 10.20511/pyr2018.v6n1.180

Al-Naggar, R. A., Osman, M., Ismail, Z., Bobryshev, Y., Ali, M. \& Menendez Gonzalez, M. (2015). Relation between type of personality and academic performance among Malaysian health sciences students. International Archives of Medicine, 182(8), 1-8. https://doi.org/10.3823/1781

Alarco, J., Aguirre-Cuadros, E., Aliaga-Chávez, Y. \& Alvarez-Andrade, E. V. (2010). Factores asociados a la realización de tesis en pregrado de Medicina en una universidad pública del Perú. Ciencia e Investigación Médica Estudiantil Latinoamericana (CIMEL), 15(2), 66-70. Recuperado de http://www.redalyc.org/articulo. oa?id $=71721155005$

Alarco, J. J., Changlio-Calle, G. \& Cahuana-Salazar, M. (2017). Investigación en pregrado: interés según sexo y ciclo académico. Educación Médica, 18(1), 67-73. https://doi.org/10.1016/j. edumed.2016.04.004

Atamari-Anahui, N., Roque-Roque, J. S., Robles-Mendoza, R. A., Nina, P. I. \& Falcón-Huancahuiri, B. M. (2015). Publicación de tesis de pregrado en una facultad de Medicina en Cusco, Perú. Revista Médica Herediana, 26(4), 217-221.

Borracci, R., Pittaluga, R., Álvarez Rodríguez, J., Arribalzaga, E., Poveda Camargo, R., Couto, J. \& Provenzano, S. (2014). Factors associated with academic success of medical students at Buenos Aires University. Medicina, 74(6), 451-456.

Caprara, G. V., Vecchione, M., Alessandri, G., Gerbino, M. \& Barbaranelli, C. (2011). The contribution of personality traits and self-efficacy beliefs to academic achievement: A longitudinal study. British Journal of Educational Psychology, 81, 78-96. https://doi.org/10.1348/2044-8279.002004 
Rasgo conciencia y actitud hacia la tesis en universitarios de una sociedad científica / Mamani-Benito et al.

Cassaretto Bardales, M. de los M. (2009). Relación entre las cinco grandes dimensiones de la personalidad y el afrontamiento en estudiantes pre-universitarios de Lima Metropolitana. (Tesis de Maestría), Universidad Nacional Mayor de San Marcos, Lima, Perú. Recuperado de http://cybertesis.unmsm.edu.pe/handle/ cybertesis/619

Cassaretto, M., Niño de Guzmán, I. \& Calderón García, A. (2003). Personalidad y rendimiento académico en estudiantes universitarios. Revista de Psicología, 21(1), 119-143.

Castro Rodríguez, Y., Sihuay-Torres, K. \& Pérez-Jiménez, V. (2017). Producción científica y percepción de la investigación por estudiantes de odontología. Educación Médica, 26(4), 1-4. https:// doi.org/10.1016/j.edumed.2016.11.001

Chamorro-Premuzic, T. \& Furnham, A. (2008). Personality, intelligence and approaches to learning as predictors of academic performance. Personality and Individual Differences, 44(7), 15961603. https://doi.org/10.1016/J.PAID.2008.01.003

Cupani, M., Garrido, S. \& Tavella, J. (2013). El Modelo de los Cinco Factores de Personalidad: contribución predictiva al rendimiento académico. Revista de Psicologia, 9(17), 67-87.

Cvetkovic-Vega, A., Inga-Berrospi, F. \& Mestas, C. A. (2016). Organizaciones científicas estudiantiles como semilleros de líderes y gestores de la investigación científica en el Perú. Acta Médica Peruana, 34(1), 70-71.

Eyong, E. I., David, B. E. \& Umoh, A. J. (2014). The Influence of Personality Trait on the Academic Performance of Secondary School Students in Cross River State, Nigeria. Journal of Humanities And Social Science (IOSR-JHSS), 19(3), 12-19.

Hakimi, S., Hejazi, E. \& Lavasani, M. (2011). The relationships between personality traits and students' academic achievement. Procedia - Social and Behavioral Sciences, 29, 836-845. https:// doi.org/10.1016/j.sbspro.2011.11.312

Hazrati-Viari, A., Tayarani Rad, A. \& Saeed Torabi, S. (2012). The effect of personality traits on academic performance: The mediating 
role of academic motivation. Procedia - Social and Behavioral Sciences, 32(2010), 367-371. https://doi.org/10.1016/j.sbspro. 2012.01.055

Hong, Z.-R. \& Li, H.-s. (2011). An Investigation of Students' Personality Traits and Attitudes toward Science. International Journal of Science Education, 33(7), 1001-1028. https://doi.org/ 10.1080/09500693.2010.524949

Komarraju, M. \& Karau, S. (2005). Personality and Individual Differences: The relationship between the big five personality traits and academic motivation. Personality and Individual Differences, 39(3), 557-567. https://doi.org/10.1016/j.paid. 2005.02.013

Laidra, K., Pullmann, H. \& Allik, J. (2007). Personality and intelligence as predictors of academic achievement: A cross-sectional study from elementary to secondary school. Personality and Individual Differences, 42(3), 441-451. https://doi.org/10.1016/j.paid. 2006.08.001

Malykh, S. (2017). The role of Personality Traits and Intelligence in Academic Achievement of Russian High School Students. Procedia - Social and Behavioral Sciences, 237(1), 1304-1309. https://doi.org/10.1016/j.sbspro.2017.02.214

Mamani Benito, O. J. \& Quinteros Zúńiga, D. (2014). Rasgos de personalidad y actitud hacia la elaboración de tesis en estudiantes del cuarto año de psicología de la Universidad Peruana Unión Filial Juliaca. Revista Cientifica de Ciencias de la Salud, 7(2), 2014-2306. http://dx.doi.org/10.17162/rccs.v7i2.220

Mayta-Tristán, P. (2016). Tesis en formato de artículo científico: oportunidad para incrementar la producción científica universitaria. Acta Médica Peruana, 33(2), 95-98.

McCrae, R.R. \& Costa, P.T. (1987). Validation of the five-factor model of personality across instruments and observers. Journal of Personality and Social Psychology, 52(1), 81-90. https://doi. org/10.1037/0022-3514.52.1.81 
McCrae, R. R. \& Costa, P.T. (2004). A contemplated revision of the NEO Five-Factor Inventory. Pergamon. https://doi.org/10.1016/ S0191-8869(03)00118-1

Mejía, C. R., Valladares-Garrido, M. J., Almanza-Mio, C. \& BenitesGamboa, D. (2018). Participación en una sociedad científica de estudiantes de medicina asociada a la producción científica extracurricular en Latinoamérica. Educación Médica, 20, 99-103. https://doi.org/10.1016/j.edumed.2017.10.014

Mejía, C., Inga-Berrospi, F. \& Mayta-Tristán, P. (2014). Titulación por tesis en escuela de Medicina de Lima, 2011: características, motivaciones y percepciones. Revista Peruana de Medicina Experimental y Salud Pública, 31(3), 509-514. https://doi. org/10.1016/j.edumed.2017.10.014

Mills, C., Heyworth, J., Rosenwax, L., Carr, S. \& Rosenberg, M. (2009). Factors associated with the academic success of first year Health Science students. Advances in Health Sciences Education, 14(2), 205-217. https://doi.org/10.1007/s10459-008-9103-9

Organización de las Naciones Unidas para la Educación, la Ciencia y la Cultura (2015). Informe de la Unesco sobre la ciencia: hacia 2030. Recuperado de https://unesdoc.unesco.org/ark:/48223/ pf0000265331

Pereyra-Elías, R., Huaccho-Rojas, J., Taype-Rondán, Á., Mejía, C. R. \& Mayta-Tristán, P. (2014). Publicación y factores asociados en docentes universitarios de investigación científica de escuelas de medicina del Perú. Revista Peruana de Medicina Experimental y Salud Pública, 31(3), 424-430. https://doi.org/10.17843/ rpmesp.2014.313.76

Rietveldt, F. \& Vera, L. (2012). Factores que influyen en el proceso de elaboración de la tesis de grado. Omnia, 18(2), 109-122. Recuperado de http://www.redalyc.org/pdf/737/73723402008.pdf

Robles-Alfaro, R., Vela-Alfaro, F., Huapaya-Huertas, O. \& ChacónTorrico, H. (2015). Relación entre el gasto en investigación y desarrollo con la producción científica en el Perú. Anales de la Facultad de Medicina, 76(4), 469-470. http://dx.doi.org/ 10.15381/anales.v76i4.11423 
Romero, E., Ángeles, L., Gómez, A. \& Sobral, J. (2002). La estructura de los rasgos de personalidad en adolescentes: El modelo de cincos factores y cinco alternativos. Psicothema, 14(1), 134-143. https://doi.org/10.2307/3539586

Ruiz, F. (2005). Relación entre la motivación de logro académico, la autoeficacia y la disposición para la realización de una tesis. Persona, 1(8), 145-170. Recuperado de http://repositorio.ulima. edu.pe/handle/ulima/2253

Smidt, W. (2015). Big Five personality traits as predictors of the academic success of university and college students in early childhood education. Journal of Education for Teaching, 41(4), 385-403. https://doi.org/10.1080/02607476.2015.1080419

Sobowale, K., Ham, S., Curlin, F. \& Yoon, J. (2017). Personality Traits Are Associated with Academic Achievement in Medical School: A Nationally Representative Study. Academic Psychiatry. https:// doi.org/10.1007/s40596-017-0766-5

Taype-Rondán, Á., Palma-Gutiérrez, E., Palacios-Quintana, M., Carbajal-Castro, C. \& Ponce-Torres, C. (2014). Producción científica estudiantil en Latinoamérica: un análisis de las revistas médicas de habla hispana indizadas en SciELO, 2011. FEM: Revista de la Fundación Educación Médica, 17(3), 171-177. https://doi.org/10.4321/S2014-98322014000300007

Taype-Rondán, Á., Carbajal-Castro, C., Arrunategui-Salas, G. \& Chambi-Torres, J. (2012). Limitada publicación de tesis de pregrado en una facultad de medicina de Lima, Perú, 2000-2009. Anales de la Facultad de Medicina, 73(6), 153-157. https://doi. org/10.15381/anales.v73i2.858

Taype-Rondán, A., Lajo-Aurazo, Y., Gutiérrez-Brown, R., ZamalloaMasías, N. \& Saldaña-Gonzales, M. (2002). Aporte de las sociedades estudiantiles en la publicación científica en Scielo-Perú, 2009-2010. Revista Peruana de Medicina Experimental y Salud Pública, 28(4). https://doi.org/10.17843/rpmesp.2011.284.439

Ticse, R., Ygreda, P. \& Samalvides, F. (2014). Publicación de los proyectos de investigación para la titulación como médicos 
especialistas de una universidad peruana, 2007-2010. Revista Peruana de Medicina Experimental y Salud Pública, 31(2), 292296. https://doi.org/36326085012

Tijdink, J. K., Bouter, L. M., Veldkamp, C. L. S., Van de Ven, P. M., Wicherts, J. M. \& Smulders, Y. M. (2016). Personality Traits Are Associated with Research Misbehavior in Dutch Scientists: A Cross-Sectional Study. PloS One, 11(9). https://doi. org/10.1371/journal.pone.0163251

Toro-Huamanchumo, C. J., Failoc-Rojas, V. E. \& Díaz-Vélez, C. (2015). Participación en sociedades científicas estudiantiles y en cursos extracurriculares de investigación, asociados a la producción científica de estudiantes de medicina humana: estudio preliminar. FEM: Revista de la Fundación Educación Médica, 18(4), 293298. https://doi.org/10.4321/S2014-98322015000500011

Vargas, G. (2018). Sociedades cientificas, ¿generan ciencia durante el pregrado? Revista Cientifica Ciencia Médica, 21(1), 66-67. Recuperado de http://www.scielo.org.bo/pdf/rccm/v21n1/ v21n1_a01.pdf

Vedel, A. \& Poropat, A. (2017). Personality and Academic Performance. https://doi.org/10.1007/978-3-319-28099-8

Verešová, M. (2015). Learning strategy, personality traits and academic achievement of university students. Procedia - Social and Behavioral Sciences, 174, 3473-3478. https://doi.org/10.1016/j. sbspro.2015.01.1021

Vern, J. (1958). Influence of Personality Traits on Academic Success. Journal of Counseling \& Developpment, 36(7), 497-500. https:// doi.org/10.1002/j.2164-4918.1958.tb01098.x

Recibido: 16 de abril, 2018

Revisado: 29 de agosto, 2018

Aceptado: 15 de marzo, 2019 